\title{
La formación humanista. Un reto ante el desarrollo de las tecnologías de la información y las comunicaciones
}

\author{
PhD. Sandra Pérez Alcolea \\ Universidad de Oriente \\ sandrap@uo.edu.cu
}

\author{
Lic. Lianne Guillén Pérez \\ Universidad de Oriente \\ Iguillen@uo.edu.ec
}

\author{
MSc. Airxa Heredia Elías \\ Universidad de Oriente \\ aheredia@uo.edu.ec
}

Palabras claves: formación profesional, formación humanista, pedagogía humanista y tecnologías de la información y la comunicación.

Recibido: 02 de mayo de 2017

Keywords: professional training, humanistic training, humanistic pedagogy and technology of information and communication.

Aceptado: 12 de junio de 2017

\section{RESUMEN}

La presencia de un mundo globalizado implica oportunidades y riesgos que para la sociedad y el individuo en particular ha de ser punto de reflexión en el proceso de educación y formación profesional. El desarrollo de las tecnologías de la información y la comunicación (TIC) como fundamento esencial de este proceso lo favorece, por lo que se requiere de un accionar global e individual del ser humano que contribuya a la solución de problemas sociales, económicos, culturales, políticos; pero, para ello se demanda de una educación que contribuya a tales propósitos, materializándose esta necesidad en una concepción de formación humanista como parte de la formación integral del profesional. El presente trabajo asume el enfoque de una Pedagogía Humanista que reconoce el papel activo del sujeto en el proceso de transformación de la realidad social, como actor principal del proceso educativo a partir de su contexto histórico, planteando como objetivo la reflexión del reto que enfrenta la formación del profesional de la educación ante el impacto cada vez mayor de las TIC en la sociedad.

\begin{abstract}
The presence of a globalized world implies opportunities and risks that for society and individual in particular has to be a point for reflection in the process of education and professional training. the development of Technology of Information and Communication (TIC) as essential foundation of this process favor it, for that it requires of a global and individual action of the human being to contribute solve such social, educational economical, cultural and politic problems; but for that it is demanded an education that contributes to such purposes, materializing need in a conception of humanistic formation as part of the integral training of the professional. The present piece of work assumes the humanistic pedagogical approach that recognizes the active role of the subject in the process of transformation of the social reality, as mail actor of the educative process starting from his historical context, stating as objective the reflection of the challenge the professional education training faces before each time greater impact of the new Technologies of Information and Communication in the society.
\end{abstract}




\section{INTRODUCCIÓN}

La formación del profesional de la educación debe responder a un amplio conocimiento de la ciencia y la tecnología, y en sus prioridades contribuir a la formación científico tecnológica, de forma que le permita ascender en su proyección social en correspondencia con las necesidades e intereses de la sociedad y resolver con independencia cognoscitiva los problemas de la práctica social; en esta dirección se hace necesario además, del conocimiento científico en la formación profesional, aquel que le permite desempeñarse como profesional, con un alto sentido de humanismo y responsabilidad ante los retos del mundo actual.

En Cuba, la educación ante los retos que encierra el desarrollo de las tecnologías de la información y las comunicaciones (TIC) asume como concepción la formación integral centrado en la formación de alto significado humano que permita comprender y actuar en beneficio de la sociedad, y que encierre valores humanos y cualidades, como la creatividad, la independencia, capacidad de evaluar la importancia de solución a los problemas, la auto-superación, y el compromiso social.

Reflexionar en esa formación humanista como parte de la formación integral del individuo desde un contexto en que el desarrollo de las TIC está produciendo cambios cada vez más significativos en el accionar del individuo, significa asumir con responsabilidad los retos que ellas imponen al proceso educativo en la sociedad.

\section{DESARROLLO}

La formación es categoría esencial de la Pedagogía, constituye un proceso social y cultural de prioridad en la educación intencionalmente orientada a adquirir conocimientos y modos de actuación, a formar cualidades y valores en el individuo que respondan al proyecto de sociedad que se construye. Los cambios en la educación, provocados por el contexto de las TIC's implican también transformaciones en la actividad científico educacional que exigen perfeccionar toda la labor investigativa y laboral de la práctica pedagógica en función de un mejor desarrollo profesional del estudiante de carreras pedagógicas.

Las TIC's aportan a la formación profesional de la educación la generalidad de ser una herramienta a través de la cual se puede acceder sin límite de tiempo y espacio a información y conocimiento que desde lo individual se apropia el estudiante para la selección y reflexión en su proceso de construcción del conocimiento científico, lo que dinamiza su proceso formativo, que requiere desde lo metodológico la orientación del estudiante en su práctica pedagógica, de forma que exteriorice una conducta en correspondencia con las exigencias de la sociedad actual, una formación humanista que permita desarrollar en el individuo la capacidad de crear, interactuar, colaborar y proyectar, potenciar el modo de actuación profesional en su formación como miembro de la sociedad. 
Con el desarrollo de las TIC's aparecen nuevas relaciones dinámicas en el proceso pedagógico, que a decir de Mendoza (2004) al señalar la práctica como mediadora de la formación inicial en el nuevo contexto, demanda de una concepción diferente en la formación, con un papel protagónico en la formación práctica del profesional y en el perfeccionamiento continuo de su actuación, lo que se complementa con una implicación del profesional en formación en su propio desarrollo. Estas relaciones devienen del uso de las TIC's como la nueva manera de acceso a la información, comunicación y cooperación, entre estudiante-docente, estudiante-estudiante, estudiante-comunidad escolar, las cuales han de estar sustentadas en una concepción humanista.

Se reconoce que la concepción humanista, en filosofía, se refiere a la dignidad y el valor del sujeto social, el valor de lo humano, es el respeto a la dignidad humana, por el bienestar y el desarrollo multilateral, por crear condiciones de vida social favorables; es preservar y desarrollar la vida, por lo que un aspecto que ha de tenerse en cuenta en la formación profesional de la educación, es precisamente la posibilidad y realidad de conservar y desarrollar la vida humana.

Comprender las exigencias que imprime la sociedad al proceso formativo del estudiante, al plantearse desde el desarrollo de las TIC's ; requiere, de la comprensión de una nueva forma de comunicación y colaboración que tiene lugar en los nuevos espacios educativos, lo cual significa nuevas formas de interacción comunicativa entre estudiantes y docentes, entre estudiantes y estudiantes comunidad estudiantil, planteando diferentes modos de ver y pensar los procesos.

El uso de las TIC's en la formación profesional establece un nuevo concepto de alfabetización, ellas demandan nuevas formas de alfabetización tecnológica; se plantea que la educación transita de una alfabetización informática entendida como conocimiento de la tecnología para crear documentos convencionales en HTML, materiales didácticos electrónicos, tutoriales, a una alfabetización digital entendida como conocimiento que garantiza realizar la comunicación, la interacción, el aprendizaje desde la virtualidad (internet, la educación virtual conocida como teleformación).

La alfabetización tecnológica no solo en el protagonismo que la tecnología ha adquirido en la vida cotidiana, sino en la necesidad de promover una actuación consciente en el uso de la información que circula y para la creación de nuevos conocimientos favorables al desarrollo de la sociedad. El uso de las TIC's imponen marcadas transformaciones en la configuración del proceso pedagógico, con cambios en los roles que han venido desempeñando los diversos actores del mismo; se asumen nuevas tareas y responsabilidades por estudiantes y docentes, los primeros tendrán que estar más preparados para la toma de decisiones y la regulación de su aprendizaje, mayor independencia cognoscitiva y, los segundos para diseñar nuevos entornos de aprendizaje al pasarse de un modelo unidireccional de formación donde ellos son los portadores fundamentales de los conocimientos, a otros más abiertos y flexibles en donde la información y el conocimiento se encuentra en grandes bases de datos compartidas por todos. 
Las TIC's incrementan el potencial de la información, permiten acceder a ellas sin límites de tiempo y espacio, lo que modifica la manera en que se produce el conocimiento, y en el proceso educativo la relación información-comunicación puede ser sincrónica y asincrónica; por ello docentes y estudiantes deben adquirir habilidades y destrezas que le permitan profesionalizarse en el uso de estos recursos pues desempeñan un papel fundamental en la transformación de la educación.

En la formación de este profesional, centrado en el estudiante, las TIC's en su implementación demandan de cambios en el rol del docente, el cual ha de asumir una nueva concepción de la presencialidad en el proceso de enseñanza aprendizaje, este más que transmisor directo ha de ser orientador del conocimiento, de enseñar a aprender, a estimular la comunicación e interactividad entre todos los participan- tes a través del trabajo en equipo, colaborativo en un nuevo espacio educativo, el espacio que estas tecnologías están ofreciendo al aprendizaje; pero para ello tendrá que aprender a enseñar desde las tecnologías y sobre todo ha de orientarse en una concepción humanista.

Para el docente se generan cambios en la formación del conocimiento científico a partir de las TIC's enmarcados en la nueva forma de comunicación y colaboración que tiene lugar en ese nuevo espacio educativo.

- Comunicación, de comunicación sincrónica (estudiante-docente donde el interlocutor está siempre presente en tiempo real) por la comunicación asincrónica en un espacio digital (comunicación mediada y en tiempo no real). Las tecnologías de la información y las comunicaciones son poderosas redes de comunicación donde el estudiante puede acceder a nuevas formas de organizar y estructurar la información. El rol del docente cambia y pasa a ser, de poseedor de la información a facilitador de esta información.

- Colaboración, se brinda mayor equidad y descentralización en la distribución de la información, permitiendo en el proceso una mayor interactividad; se puede participar, elegir y tomar información, pero también se puede brindar; pues la red democratiza la información (Intranet, Internet), se fomenta la búsqueda, el diálogo, el trabajo en equipo, la constancia, la curiosidad, el intercambio de los resultados de investigación, fomenta cambios en las relaciones personales, modifica las formas de interactuar y comunicarse el docente y el estudiante, los docentes y los estudiantes entre sí favoreciendo el intercambio de conocimientos, el debate y la reflexión colectiva.

Estas posibilidades que ofrecen las TIC's son elementos modificadores de la cultura y educación del individuo, significan reconocer en su uso como mecanismo de comunicación y colaboración, pues a través de sus redes se favorece la creación de patrones tecno-culturales, entendidos estos como el conjunto de formas aprendidas en el interactuar con las tecnologías y que se reproducen en la práctica pedagógica del estudiante como la nueva forma de acceder, compartir, comunicar, crear información y conocimiento y que se incorporan a la cultura del contenido de la enseñanza. 
Las TIC's cambian las formas de cómo hacer las cosas, escribir, leer, almacenar, por nuevas formas de trabajar y comunicar; se garantiza un mayor alcance al desarrollo individual del estudiante, son herramientas poderosas de apoyo, materiales didácticos motivadores, socializadores que potencian las habilidades de comunicación en el proceso de enseñanza.

La presencia de las TIC's, en la formación profesional de la educación constituye una poderosa red de comunicación que integran tecnologías, servicios y aplicaciones, donde docentes y estudiantes pueden acceder a nuevas formas de organizar y estructurar la información, fomentar la búsqueda, el diálogo, el trabajo en equipo, el intercambio de resultados investigativos, producir, ordenar, compactar y movili- zar cantidades ilimitadas de información, interactuar y comunicarse favoreciendo el intercambio de conocimiento, que desde una concepción humanista promueva el desarrollo de la sociedad y el compromiso del estudiante en su práctica pedagógica.

El reconocer los cambios que produce el uso de las TIC's en la educación, si se observa la situación actual caracterizada por una fuerte relación y complementa- ción entre tecnología y enseñanza, donde las tecnologías condicionan modelos que ayudan a plantear la enseñanza bajo una nueva perspectiva; los nuevos medios tecnológicos logran no solo cambiar los sistemas de evaluación hombre-medio, sino también instalarse como componente cultural, por ello su desarrollo, actúa como eje dinamizador en la formación del conocimiento científico pedagógico necesario para los estudiantes de carreras pedagógicas.

Si se asumen estos cambios que proporciona el contexto de las TIC's al proceso de enseñanza y aprendizaje, en la formación del conocimiento científico pedagógico en su dinámica integradora, será necesario tomar en cuenta:

- $\quad$ El dominio de las habilidades de la informática, para instrumentar el trabajo interactivo, la comunicación, el intercambio, la reflexión en el contenido de la enseñanza entre estudiantes y docentes sin que medie espacio y tiempo.

- Dominar procedimientos informáticos básicos que permitan crear nuevos espacios educativos con el uso de estos medios y recursos, de forma novedosa que motive el aprendizaje en los estudiantes.

- $\quad$ Adquirir las destrezas técnicas esenciales para acceder y procesar gran volumen de información y conocimientos que puede encontrar a través de Internet.

- $\quad$ Evaluar la calidad de la información encontrada en las páginas web.

- $\quad$ Determinar estrategias que faciliten el tratamiento del contenido desde la virtualidad. (intencionalidad formativa).

- $\quad$ Conocer el valor que adquiere en el proceso de formación del conocimiento científico el aprovechamiento óptimo de las nuevas oportunidades derivadas del contexto de las tecnologías de la información y las comunicaciones. 
- $\quad$ Comprender los retos que imponen las tecnologías de la información y las comunicaciones para la formación permanente, en función del logro de la independencia cognoscitiva.

- Coadyuvar a la formación de valores ante las nuevas vías para compartir, adquirir, crear conocimientos, a favor de la solidaridad, la ética profesional, y el sentido de pertenencia a una sociedad humanista.

En este sentido se concuerda con Arana, Batista y Ramos (2003) al plantear que:

El humanismo es sencillamente un intento y una actitud del espíritu humano en pernente proceso de evolución, que se inicia en el momento en que tomamos conciencia de nuestra diferencia con las demás especies biológicas, que busca el respeto, la dinidad y los derechos del ser humano para la formación integral de su individualidad y de su personalidad, lo cual requiere y conlleva condiciones sociales que propicien su transformación y realización como ser humano.

Desde el punto de vista filosófico se suele entender como humanismo, a todo aquel movimiento intelectual que considere como fundamental la naturaleza humana y los intereses del hombre. El humanismo debe considerarse como una actividad permanente que debe tender a realizar, en el hombre, el ideal de hombre, situar al hombre como valor principal en todo lo existente, concebir al hombre como fin y no como medio.

En el contexto de las TIC's, el estudiante significa lo que realmente contribuye al desarrollo de su práctica pedagógica, comunica e intercambia con el colectivo, aspecto que orientado en su desarrollo por el docente desde una posición intencionada de carácter formativo de acuerdo al objeto social del estudiante, definido en el modelo del profesional de carreras pedagógicas, favorece el desarrollo individual y colectivo en la identificación y solución de los problemas profesionales.

En la Pedagogía Humanista se reconoce el papel activo del sujeto en el proceso de transformación de la realidad social a partir de su contexto histórico, se complementa con la concepción de formación integral centrado en la formación de alto significado humano que permita comprender y actuar en beneficio de la sociedad, por lo que encierra cualidades a lograr en el profesional como la creatividad, la independencia, la auto-superación acompañado del compromiso social, sustento y esencia en el modelo pedagógico del profesional de la educación en Cuba.

Se responde de este modo a la comprensión axiológica en la formación del conocimiento científico del estudiante, y se da en la relación entre educación y valores, estos últimos como mecanismo regulador de la conducta del individuo en un contexto social en que ha de tomar en cuenta no solo intereses individuales, sino sociales, pues a través de los valores el sujeto se autoconoce, conoce sus necesidades y la posibilidad de satisfacerlas o proyecta condiciones ideales para ello, este auto-reconocimiento del individuo se realiza a partir de identificar su lugar y papel en la solu- 
ción de los problemas de la práctica pedagógica, en este caso en correspondencia con el desarrollo tecnológico en un nuevo contexto social.

Concebir una dinámica integradora entre el desarrollo de una formación científico tecnológica sustentada en una Pedagogía Humanista a través del carácter transformador del conocimiento científico en el contexto formativo de las TIC's, como proceso sistematizador, se genera el ascenso de lo abstracto a lo concreto en la construcción y reconstrucción del conocimiento científico en el profesional de la educación. Esta relación dialéctica es expresión de la relación externa del proceso de formación del conocimiento científico en el contexto de desarrollo de estas tecnologías como representación de las exigencias de la educación en la época actual, en el vínculo sociedad-educación, lo cual fundamenta los cambios que se operan en la relación dialéctica interna del proceso educación-formación-aprendizaje desde la formación-desarrollo en la Pedagogía.

En esta dirección la formación científica tecnológica en el estudiante de carreras pedagógicas debe responder a:

- Desarrollar una actitud crítica, reflexiva e investigativa en relación con los problemas que emanen del desarrollo de las TIC's en el marco del proceso de la práctica pedagógica.

Comunicar, compartir ideas y proyectar decisiones del proceso de la práctica pedagógica resultado del trabajo docente, metodológico, investigativo y laboral, desde el contexto de las TIC's.

El carácter transformador del conocimiento científico desde el contexto de las TIC's , concebido bajo una intencionalidad formativa, contribuye al desarrollo de la capacidad transformadora humana, creando los espacios para ello, Fuentes, H. (2009); lo que reafirma en el estudiante concepciones tecno-científicas, tecno-transformadoras, como parte de los fundamentos de la construcción del conocimiento científico pedagógico, que requiere la Pedagogía Humanista actual, concebir una educación que desde el desarrollo de la ciencia y la tecnologías garantice la transformación social en beneficio del desarrollo del individuo en la correlación de lo individual y lo social.

Acceder, estructurar, ordenar, compartir, crear conocimiento desde el contexto de las TIC's es hoy una necesidad en el proceso formativo del estudiante de carreras pedagógicas para garantizar los cambios de prácticas, comportamientos y aprendizajes en el proceso de enseñanza y asegurar desde el sustento de la Pedagogía Humanista un estudiante comprometido con la práctica social.

Los problemas del mundo de hoy agravados con el acelerado proceso de globalización requieren de profesionales cada vez más comprometidos con los cambios sociales a favor del desarrollo humano. Hoy se exige de profesionales con capacidad no solo de comunicarse coherentemente con los demás, sino además de una clara concepción del mundo, la sociedad y de sí mismo, de capacidad de pensar reflexi- 
vamente a partir de principios morales, éticos, de ser creativos, capaz de manejar gran cantidad de información, resolver problemas y trabajar en colectivo, por ello es necesario de una adecuada labor educativa, en valores pues el educador tendrá que asumir una posición dialéctica, crítica e innovadora que permita en cualquier nivel de enseñanza transformar al sujeto que aprende, a la realidad social y a sí mismo, es una labor con una intencionalidad que se erige en principio como contenido de la educación para la educación en valores.

En el contexto de las TIC's, una formación en valores reconoce a la necesidad de asumir de forma comprometida un modo de actuación que responda a las exigencias de transformación de la sociedad, por lo que deberá tomarse en cuenta los espacios con que cuenta el docente para su labor educativa:

- Pedagógico

- Social

- Cultural

-Investigativa

Pedagógico. En esta dirección hay que ver a la informática como herramienta de apoyo, material dinámico del profesor, como elemento motivador en el proceso de enseñanza-aprendizaje. Las computadoras permiten la recopilación, el procesamiento y la producción automatizada de datos y conocimiento, ella pasa a ser un instrumento de trabajo fundamental.

Por ello los docentes deben adquirir nuevas habilidades y destrezas que le permiten profesionalizarse en el uso de estos recursos pues esta tecnología ha comenzado a jugar un papel fundamental en toda la transformación educativa, pensar informáticamente supone operaciones mentales distintas y por tanto requiere de propuestas pedagógicas específicas. Al respecto hay muchas investigaciones relacionadas con su efectividad y transformación en el proceso de enseñanza yaprendizaje.

Social. Refleja los vínculos personales, la nueva manera de comunicación humana que elimina la distancia. Hoy esta tecnología exige de la educación adaptación a nuevas formas de acceso a la información que condicionan nuevos modos de expresión que supone a su vez nuevas formas de acceso y modelos de participación y recreación cultural, condicionando cambios en el sistema de relaciones alumno- profesor, entre los alumnos y entre profesores.

En esta dirección se debe fomentar el desarrollo de habilidades que fortalezcan las relaciones de colaboración, pues el uso de las computadoras favorece el trabajo investigativo, el intercambio de conocimiento, el debate y la reflexión de problemas educativos, sociales, fomentan la preparación del estudiante en su desempeño profesional. Asume la necesidad de fomentar la responsabilidad colectiva en el uso del conocimiento y la información en beneficio de la transformación social. 
Cultural. Se le atribuye al acelerado desarrollo que alcanza las TIC's y su presencia de todas las esferas de la sociedad. Se habla hoy que ellas están provocando cambios y mejoras en el conjunto de la sociedad. La creación de redes en la educación facilita una mayor socialización del conocimiento y por tanto un mayor acceso a la cultura. Su utilización profundiza la relación objetiva entre individuo- cultura y enseñanza. En esta dirección la educación ha de favorecer la aplicación y utilización cada vez más de las TIC's en la solución de problemas como acceso, manejo, distribución y procesamiento de la información y el conocimiento como herramienta esencial en un proceso transformador.

Investigativa. El profesor incluye en sus funciones la investigación, desde esta dirección el desarrollo tecnológico en la información encierra en sí misma un proceso de investigación, pues en su desempeño el docente debe buscar (investigar) no solo como hacer mejor las cosas sino como hacer aquellas que no podemos hacer y que las nuevas tecnologías las están proporcionando.

\section{CONCLUSIONES}

La Pedagogía Humanista debe estar dirigida a la formación de estudiantes en el humanismo como valor moral, el cual se manifiesta en la manera de formar nuevos valores epistemológicos y ético-filosóficos en el proceso de enseñanza y aprendizaje en general, es una integración del conocimiento-valores-valoraciones, al entender los valores como eje entre los componentes de este proceso en que interviene lo epistemológico y los valores.

La formación humanista en el contexto de las tecnología de la información y las comunicaciones es requerimiento fundamental que precisa desarrollar el estudiante, si se aspira a un profesional que sea capaz de transformar la práctica pedagógica en correspondencia con las exigencias del desarrollo científico tecnológico, por lo que será necesario propiciar el desarrollo de una práctica docente investigativa integrada al manejo de las TIC's, de forma que responda a las transformaciones de la educación en el contexto actual y la realidad social.

\section{REFERENCIAS BIBLIOGRÁFICAS}

Addine, F. (1995). Exigencia en la formación del profesional pedagógico. Pedagogía 95. La Habana.

Agustín, L. (2013). La Economía del Conocimiento y el Socialismo. La Habana: Editorial Academia.

Alphonse, M. (2012). Benefits of Educational Technology. (en línea). Disponible en: http://merseamusic.blospot.com. [Consultado el 20 dejunio del 2014]

Arana, M., Batista, N., \& Ramos Castro, A. ( 2003). Los valores en el desarrollo de competencias profesionales. En monografía virtuales, ciudadanía, democracia y valores en sociedades plurales. 
Arana, M., \& Armenteros, J. (s.f.). Los Valores en la Formación Profesional. Universidad Colegio Mayor de Cundinamarca.

Area , M. (2009). Introducción a la tecnología educativa. España: Universidad de La Laguna.

Báxter, E. (2003). Cuando y cómo educar en valores. La Habana: Pueblo y Educación.

Berizzo, C. (2013). Las tecnologías de la información y las comunicaciones como herramienta de enseñanza aprendizaje en las prácticas pedagógicas. XV Congreso Internacional de Informática en la Educación. Informática 2013. La Habana.

Blanco, L. (2013). Aspectos morales y éticos de las TIC. Revista Temas (74), 30-37.

Blanco, J., \& Encinosa, L. (2013). Aspectos morales y éticos de las TIC. Revista Temas (74), págs. 30-37.

Castellanos Simons, B. (s.f.). La visión humanista acerca de la educación y el aprendizaje. La Habana: Maestría en Educación. Módulo teorías del aprendizaje Material de consulta.

Cortón, B. (2007). Humanidades y Humanismo, formación humanista y labor ideopolítica del docente. Stgo de Cuba: Soporte Digital.

Fabelo, J. R. (2007). Los valores y sus desafíos actuales. Nueva Cultura.

Fuentes, H. (2008). La formación de los profesionales en la contemporaneidad. Concepción científica Holística Configuracional en la Educación Superior. CeeS Manuel F. Gran. Universidad de Oriente. Material digitalizado.

Fuentes, H., Montoya, J., \& L., F. (s.f.). La construcción del conocimiento científico en las ciencias pedagógica. Stgo de Cuba: Catedra Manuel F. Gran.

Horruitiner, P. (2006). La Universidad Cubana: el modelo de formación. La Habana: Félix Varela.

Horruitiner, P. (2011). La educación superior. Retos y perspectivas en la sociedad cubana. Curso \# 17 Pedagogía 2011. La Habana.

Horruitiner, P. (2013). El modelo de formación de la nueva Universidad Cubana. Conferencia en Pedagogía 2013. La Habana.

Pérez, S. (2015) Dinámica integradora de la formación del conocimiento científico pedagógico en el contexto de las tecnologías de la información y las comunicaciones. Tesis Doctoral 